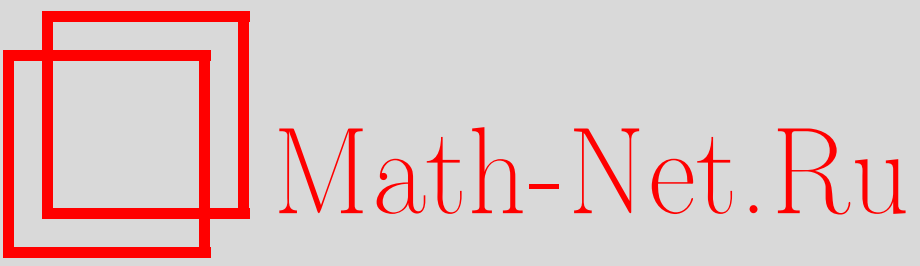

А. И. Эстеров, Индексы 1-форм, результанты и многогранники Ньютона, УМH, 2005, том 60, выпуск 2, 181-182

DOI: https://doi.org/10.4213/rm1425

Использование Общероссийского математического портала Math-Net.Ru подразумевает, что вы прочитали и согласны с пользовательским соглашением

http://www.mathnet.ru/rus/agreement

Параметры загрузки:

IP : 54.84 .234 .179

26 апреля 2023 г., 18:09:53 


\title{
ИНДЕКСЫ 1-ФОРМ, РЕЗУЛЬТАНТЫ И МНОГОГРАННИКИ НЬЮТОНА
}

\author{
А. И. ЭСТЕРОВ
}

В этой работе индекс пересечения алгебраического многообразия $R \subset \mathbb{C}^{m}$ специального вида с образом ростка аналитического отображения $F:\left(\mathbb{C}^{n}, 0\right) \rightarrow\left(\mathbb{C}^{m}, 0\right)$ выражается через многогранники Ньютона компонент $F$ при условии, что главные части компонент находятся в общем положении. Частными случаями индексов пересечения такого типа являются различные обобщения индекса Пуанкаре-Хопфа нуля векторного поля на случай 1-форм на особых многообразиях (например, индекс Гусейн-Заде-Эбелинга [1]). В качестве следствия получается также описание многогранника Ньютона многомерного результанта, обобщающее результаты из [2] и [3].

Определим "относителшный" вариант смешанного объема многогранников, необходимьй для формулировки результата. Будем говорить, что выпуклый (не обязательно ограниченный) многогранник $A \subset \mathbb{R}^{n}$ похож на конус с вершиной $\Gamma \subset \mathbb{R}^{n}$, если $A=\Gamma+A^{\prime}$, где $A^{\prime} \subset \mathbb{R}^{n}-$ ограниченньй многогранник. Рассмотрим множество $\mathscr{M}_{\Gamma}$ всех упорядоченных пар многогранников $(A, B)$, похожих на конус $\Gamma \subset \mathbb{R}^{n}$, таких что симметрическая разность $A \triangle B$ ограничена. Это множество - полугруппа относительно сложения пар $(A, B)+(C, D)=(A+C, B+D)$.

ОПредЕЛЕНИЕ. Объемом $V(A, B)$ пары многогранников $(A, B) \in \mathscr{M}_{\Gamma}$ назовем разность объемов множеств $A \backslash B$ и $B \backslash A$. Смешанным объемом пар многогранников называется симметричная полилинейная функция $\operatorname{Vol}_{\Gamma}: \underbrace{\mathscr{M}_{\Gamma} \times \cdots \times \mathscr{M}_{\Gamma}}_{n} \rightarrow \mathbb{R}$ такая, что $\operatorname{Vol}_{\Gamma}((A, B), \ldots,(A, B))$ $=V(A, B)$ для любой пары $(A, B) \in \mathscr{M}_{\Gamma}$.

Доказательство существования и единственности, основные свойства и способы вычисления смешанного объема многогранников легко переносятся на смешанный объем пар. Для множества $\Sigma \subset \mathbb{Z}^{n}$ обозначим через $\mathbb{C}[\Sigma]$ множество многочленов Лорана $\left\{\sum_{a \in \Sigma} c_{a} t^{a} \mid c_{a} \in \mathbb{C}\right\}$.

ОПредЕлЕниЕ. Для конечных множеств $\Sigma_{i} \subset \mathbb{Z}^{N}, i=1, \ldots, I$, замькание множества

$$
\left\{\left(\varphi_{1}, \ldots, \varphi_{I}\right) \mid \varphi_{i} \in \mathbb{C}\left[\Sigma_{i}\right], \exists t \in(\mathbb{C} \backslash\{0\})^{N}: \varphi_{1}(t)=\cdots=\varphi_{I}(t)=0\right\} \subset \mathbb{C}\left[\Sigma_{1}\right] \oplus \cdots \oplus \mathbb{C}\left[\Sigma_{I}\right]
$$
назовем результантным многообразием $R\left(\Sigma_{1}, \ldots, \Sigma_{I}\right)$.

В обозначениях этого определения рассмотрим росток голоморфного отображения

$$
\begin{aligned}
& F:\left(\mathbb{C}^{n}, 0\right) \rightarrow\left(\mathbb{C}\left[\Sigma_{1}\right] \oplus \cdots \oplus \mathbb{C}\left[\Sigma_{I}\right], R\left(\Sigma_{1}, \ldots, \Sigma_{I}\right)\right), \\
& F(x)=\left(\sum_{a \in \Sigma_{1}} f_{(a, 1)}(x) t^{a}, \ldots, \sum_{a \in \Sigma_{I}} f_{(a, I)}(x) t^{a}\right),
\end{aligned}
$$

заданный ростками $f_{(a, i)}: \mathbb{C}^{n} \rightarrow \mathbb{C}, i=\{1, \ldots, I\}, a \in \Sigma_{i}$, где $n=\operatorname{codim} R\left(\Sigma_{1}, \ldots, \Sigma_{I}\right)$. В случае общего положения главных частей ростков функций $f_{(a, i)}$ индекс пересечения

$$
F\left(\mathbb{C}^{n}\right) \circ R\left(\Sigma_{1}, \ldots, \Sigma_{I}\right)
$$

выражается через многогранники Ньютона $\Delta_{f_{(a, i)}}$ функций $f_{(a, i)}$. Для точной формулировки введем следующие обозначения. Пусть $\operatorname{Conv}(A)$ - выпуклая оболочка множества $A, \mathbb{R}_{+}^{n} \subset \mathbb{R}^{n}$ - положительный октант. Рассмотрим многогранники $\Delta_{i}=\operatorname{Conv}\left(\Sigma_{i}\right) \times \mathbb{R}_{+}^{n} \subset \mathbb{R}^{N} \oplus \mathbb{R}^{n}$ и содержащиеся в них $\widetilde{\Delta}_{i}=\operatorname{Conv}\left(\bigcup_{(i, a), a \in \Sigma_{i}}\{a\} \times \Delta_{f_{(a, i)}}\right) \subset \mathbb{R}^{N} \oplus \mathbb{R}^{n}$. Через $\operatorname{Lin}\left(A_{1}, \ldots, A_{J}\right)$ обозначим минимальную подрешетку в $\mathbb{Z}^{N}$, в которую параллельными переносами помещаются множества $A_{i} \subset \mathbb{Z}^{N}, i=1, \ldots, J$.

Работа частично поддержана грантами РФФИ-04-01-00762 и НШ-1972.2003.1. 
ОПРЕДЕЛЕниЕ. Набор множеств $\Sigma_{i} \subset \mathbb{Z}^{N}, i=1, \ldots, I$, будем назьвать существенным, если $J-\operatorname{rk} \operatorname{Lin}\left(\Sigma_{i_{1}}, \ldots, \Sigma_{i_{J}}\right)<I-N$ для любого поднабора $\left\{i_{1}, \ldots, i_{J}\right\} \subset\{1, \ldots, I\}$ и $\operatorname{Lin}\left(\Sigma_{1}, \ldots, \Sigma_{I}\right)=\mathbb{Z}^{N}$.

ТЕОремА. Пусть в обозначениях, введенных выше, $\Sigma_{i} \subset \mathbb{Z}^{N}, i=1, \ldots, I,-$ существенный набор конечных множеств и множества $\left(\Delta_{i} \backslash \widetilde{\Delta}_{i}\right), i=1, \ldots, I$, ограничены. Тогда:

1) $\operatorname{codim} R\left(\Sigma_{1}, \ldots, \Sigma_{I}\right)=I-N$;

2) если главные части ростков функций $f_{(a, i)}: \mathbb{C}^{n} \rightarrow \mathbb{C}, a \in \Sigma_{i}$, в общем положсении, то индекс пересечения $(*)$ определен и равен $I$ ! $\mathrm{Vol}_{\{0\} \times \mathbb{R}_{+}^{n}}\left(\left(\Delta_{1} \backslash \widetilde{\Delta}_{1}\right), \ldots,\left(\Delta_{I} \backslash \widetilde{\Delta}_{I}\right)\right)$;

3) в общем случае индекс (*) не меньше указанного значения или не определен.

Теорема сформулирована для случая, когда набор $\Sigma_{i} \subset \mathbb{Z}^{N}, i=1, \ldots, I$, существенный, так как общий случай легко сводится к этому. Доказательство основано на технике торических разрешений Хованского [4]. Пункт 1) в случае $I=N+1$ доказан в [3]. Приведем теперь примеры приложений этого резултата.

1. Пусть $f_{i}:\left(\mathbb{C}^{n}, 0\right) \rightarrow(\mathbb{C}, 0), i=1, \ldots, k,-$ ростки голоморфных функций и $\omega=\sum_{j=1}^{n} \omega_{j} d x_{j}$ - росток голоморфной 1-формы на $\left(\mathbb{C}^{n}, 0\right)$. Рассмотрим отображение

$$
F=\left(\left(\omega, d f_{1}, \ldots, d f_{k}\right),\left(f_{1}, \ldots, f_{k}\right)\right):\left(\mathbb{C}^{n}, 0\right) \rightarrow\left(\mathbb{C}^{n \times(k+1)} \oplus \mathbb{C}^{k}, R \times\{0\}\right),
$$

где $\left(\omega, d f_{1}, \ldots, d f_{k}\right)$ - матрица размера $n \times(k+1)$ с голоморфными коэффициентами и $R \subset$ $\mathbb{C}^{n \times(k+1)}$ - множество вырожденных матриц в пространстве матриц размера $n \times(k+1)$. Заметим, что $\left(\mathbb{C}^{n \times(k+1)} \oplus \mathbb{C}^{k}, R \times\{0\}\right)=\left(\mathbb{C}\left[\Sigma_{1}\right] \oplus \cdots \oplus \mathbb{C}\left[\Sigma_{I}\right], R\left(\Sigma_{1}, \ldots, \Sigma_{I}\right)\right)$, где $I=n+k$, $\Sigma_{1}=\cdots=\Sigma_{n}-$ стандартный симплекс в $\mathbb{Z}^{k}$ и $\Sigma_{n+1}=\cdots=\Sigma_{n+k}-$ точка в $\mathbb{Z}^{k}$. Легко видеть, что в этом случае индекс пересечения $(*)$ равен индексу Гусейн-Заде-Эбелинга ограничения 1- формы $\omega$ на полное пересечение $\left\{f_{1}=\cdots=f_{k}=0\right\}$, определенному в [1] (в частности, эти две величины корректно определены одновременно). Таким образом, сформулированная вьше теорема позволяет вычислять индекс Гусейн-Заде-Эбелинга в терминах многогранников Ньютона ростков функций $f_{i}, i=1, \ldots, k$, и компонент 1 -формы $\omega_{j}, j=1, \ldots, n$.

2 . Пусть $I=N+1$ в условиях сформулированной выше теоремы, тогда неприводимая гиперповерхность $R\left(\Sigma_{1}, \ldots, \Sigma_{I}\right)$ задается неприводимым многочленом, которьй называется обобщенным результантом. Для ростка отображения $F:\left(\mathbb{C}^{1}, 0\right) \rightarrow\left(\mathbb{C}\left[\Sigma_{1}\right] \oplus \cdots \oplus \mathbb{C}\left[\Sigma_{I}\right], 0\right)$ с компонентами $f_{(a, i)}=\sum_{j=k_{(a, i)}}^{\infty} c_{j,(a, i)} s^{j}$, где $a \in \Sigma_{i}, k_{(a, i)} \subset \mathbb{N}$ и числа $c_{k_{(a, i)},(a, i)}$ в общем положении, индекс пересечения $(*)$ равен значению опорной функции многогранника Ньютона обобщенного резултанта на ковекторе с компонентами $k_{(a, i)}$. Таким образом, сформулированная вьше теорема дает описание этого многогранника Ньютона.

Я благодарен С. М. Гусейн-Заде за постановку задачи и постоянное внимание к работе, а также А. Г. Хованскому за полезные обсуждения.

\section{СПИСОК ЛИТЕРАТУРЫ}

[1] W. Ebeling, S. M. Gusein-Zade // Mosc. Math. J. 2003. V. 3. № 2. P. 439-455. [2] I. M. Gel'fand, M. M. Kapranov, A. V. Zelevinsky. Discriminants, Resultants, and Miltidimensional Determinants. Boston: Birkhäuser, 1994. [3] B. Sturmfels // J. Algebraic Combin. 1994. V. 3. № 2. Р. 207-236. [4] А. Г. Хованский // Функц. анализ и его прил. 1977. Т. 11. № 4. C. 56-67.

Московский государственный университет им. М. В. Ломоносова
Представлено С.М. Гусейн-Заде Принято редколлегией 25.01.2005 